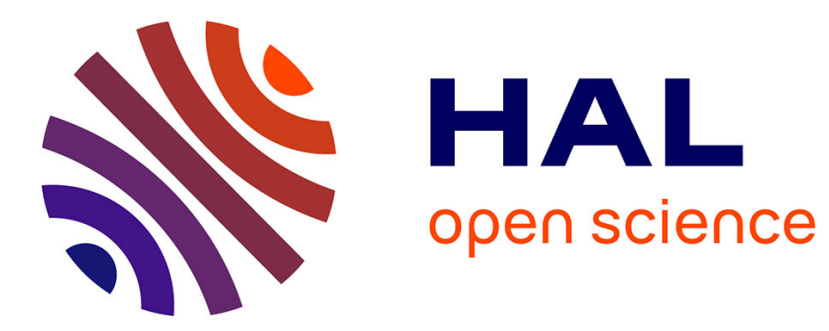

\title{
Leaky waveguide modes along the edge of a dielectric wedge
}

\author{
T. Maletzky, K. Tanaka, T. Grosjean, U.C. Fischer
}

\section{To cite this version:}

T. Maletzky, K. Tanaka, T. Grosjean, U.C. Fischer. Leaky waveguide modes along the edge of a dielectric wedge. Optics Letters, 2009, 34 (12), pp.1909-1911. 10.1364/OL.34.001909 . hal-00455129

\section{HAL Id: hal-00455129 \\ https://hal.science/hal-00455129}

Submitted on 18 Apr 2021

HAL is a multi-disciplinary open access archive for the deposit and dissemination of scientific research documents, whether they are published or not. The documents may come from teaching and research institutions in France or abroad, or from public or private research centers.
L'archive ouverte pluridisciplinaire HAL, est destinée au dépôt et à la diffusion de documents scientifiques de niveau recherche, publiés ou non, émanant des établissements d'enseignement et de recherche français ou étrangers, des laboratoires publics ou privés. 


\title{
Leaky waveguide modes along the edge of a dielectric wedge
}

\author{
Tobias Maletzky, ${ }^{1,4}$ Kazuo Tanaka, ${ }^{2,5}$ Thierry Grosjean, ${ }^{3,6}$ and Ulrich C. Fischer ${ }^{1,3, *}$ \\ ${ }^{1}$ Physikalisches Institut, Westfälische Wilhelms-Universität, 48149 Münster, Germany \\ ${ }^{2}$ Department of Electronics and Computer Engineering, Gifu University, Yanagido 1-1, Gifu 501-11, Japan \\ ${ }^{3}$ Franche-Comté Electronique Mecanique Thermique et Optique-Sciences et Technologies, \\ Laboratoire d'Optique, CNRS, Université de Franche-Comté, 25030 Besançon CEDEX, France \\ ${ }^{4}$ e-mail: t.maletzky@gmx.de \\ ${ }^{5}$ e-mail: tanaka@tnk.info.gifu-u.ac.jp \\ ${ }^{6}$ e-mail: thierry.grosjean@univ-fcomte.fr \\ *Corresponding author: fischeu@uni-muenster.de
}

\begin{abstract}
It is shown experimentally that a Gaussian beam focused from within a finite wedge onto its edge at an angle of total reflection excites a leaky waveguide mode propagating along the edge. This newly described phenomenon is interpreted in terms of waveguide modes of a tapered dielectric slab of a mode index, which is known to decrease with slab thickness. This decrease in the refractive index leads to a gradual refraction of the incident beam parallel to the edge. A numerical simulation of a focused Gaussian beam incident on a finite dielectric wedge gives a full account of this phenomenon.
\end{abstract}

We consider a beam of light incident on a dielectric wedge structure at an oblique angle with respect to the edge of the wedge as shown schematically in Fig. 1. For a plane wave incident on an infinitely extended wedge at an angle of incidence $\varepsilon$ with respect to the edge, which is larger than the critical angle of total reflection of the dielectric material, the plane wave will be totally reflected in the wedge structure. For a focused Gaussian beam of a finite cross section, a similar phenomenon may be expected as the well known Goos-Hänchen shift [1], which occurs for the total reflection of a Gaussian beam at a dielectric interface. The underlying phenomenon to the GoosHänchen shift is the so-called lateral wave, which can be considered as a leaky surface mode at the dielectric-air interface. It is shown here that a focused Gaussian beam incident on a finite dielectric wedge at an angle of total reflection is gradually deflected to be eventually aligned parallel to the edge. This phenomenon can be understood qualitatively in terms of a model of the wedge as a tapered slab with an effective refractive index decreasing with slab thickness [2] as a gradual refraction of the incident beam parallel to the edge. In the experimental part we present evidence for the leaky waveguide modes by observing light emission from the tip of a finite wedge (see Fig. 1) for a condition where a Gaussian beam is focused into the wedge at a location remote from the tip. Finally, numerical calculations simulate a Gaussian beam incident on the wedge.

The experimental configuration is shown in Fig. 2. As a wedge structure with an edge with a radius of curvature of the order of $5 \mathrm{~nm}$ [3], we use a glass fragment, which is formed by the cleaving of a piece of cover glass twice at a right angle [4]. The fragment is mounted on a prism serving to couple light into the tip. A twin microscope is used for the excitation and the observation of the leaky waveguide mode. A white-light source illuminates a circular iris dia- phragm. A demagnified image of the diaphragm is projected into the wedge, which is in focus at the edge. The beam, which is reflected from the edge, is reflected from the interface of the glass fragment opposite to the edge, and a magnified image of the edge is formed in the image plane, which is detected by a CCD camera. The NA of the incident beam and the angle of incidence of the beam can be adjusted by an adjustable iris diaphragm in the diffraction plane of the illumination pathway. With the second microscope, an image of the tip or the edge is formed from the outside of the wedge.

The experimental results are shown in Fig. 3. Figure 3(a) shows the reflected image of the edge for conditions of total reflection of the incident beam. The NA of the illuminating beam was adjusted such that the beam was totally reflected in the wedge and no light was emitted from the edge to the outside

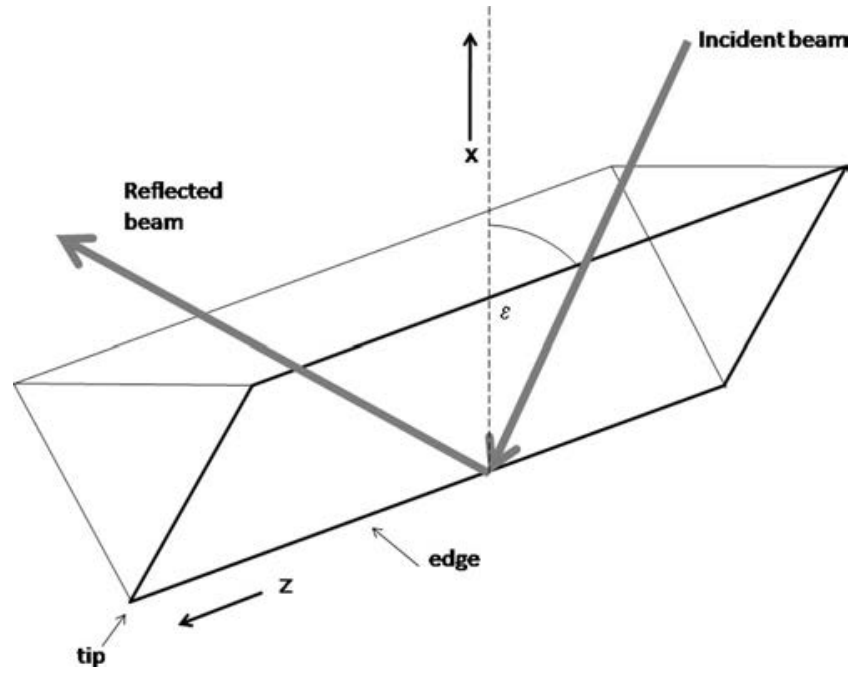

Fig. 1. Schematic view of a beam of light incident at an angle $\varepsilon$ of total reflection onto the edge of a finite wedge, which ends at a tip. 


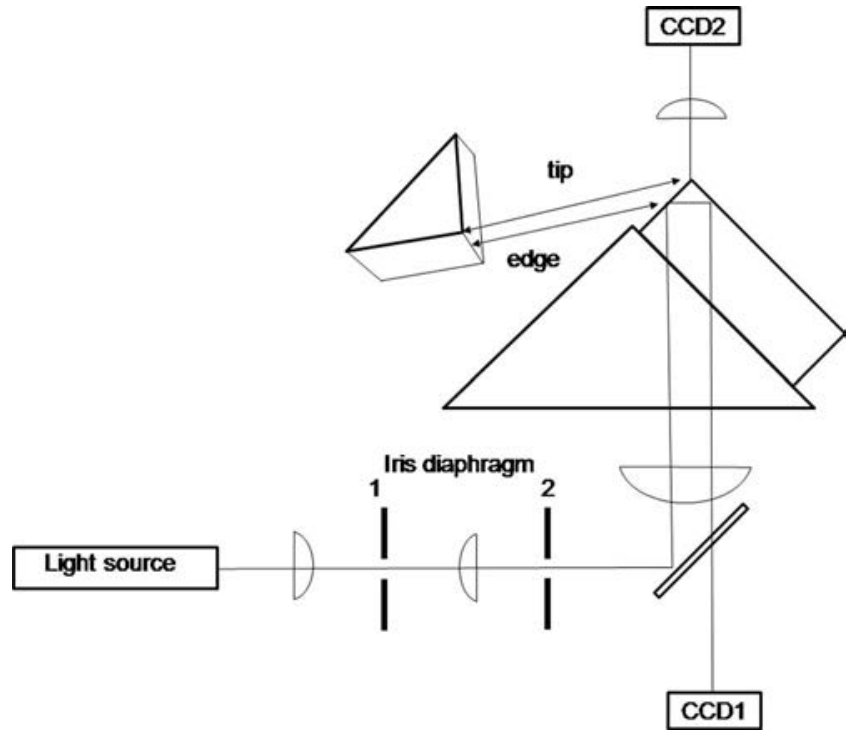

Fig. 2. Experimental configuration for the observation of a leaky waveguide mode in a wedge structure. A triangular glass fragment serves as a wedge structure. It is attached to a prism such that a focused beam of light can be injected into the edge of the wedge by using a microscope. The beam reflected from the edge is observed by a CCD1 camera. The CCD2 camera attached to a second microscope is used to detect light, which is emitted from the tip of the wedge.

[Fig. 3(d)]. Figure 3(b) shows the light emitted from the edge as observed with the upper microscope when it is focused on the edge and when the incident beam has a large NA such that parts of the incident beam with a high NA are not totally reflected and lead to radiation from the edge. Light emission from the edge is clearly observed. Adjusting the focus such that the tip is in focus, a blurred image of the emission from the edge is observed and, additionally, somewhat separated from the emission from the edge, a weak emission from the tip is seen [Fig. 3(c)]. The NA was then decreased by reducing the diameter of the iris diaphragm in the illumination pathway until the radiative emission of the edge disappeared, but the emission from the tip still prevails [Fig. 3(d)]. This was also the condition for recording Figs. 3(a) and 3(e). In Fig. 3(e) the structure of the tip is visible by an additional weak delocalized background illumination. The arrangement of the tip is indicated in Fig. 3(f). These results can be interpreted by a leaky waveguide mode propagating parallel to the edge. This leaky waveguide mode is optically excited in the wedge structure by the Gaussian beam incident onto the edge at an angle of total reflection, which is directed into the wedge at a location remote from the tip. At the tip apex the waveguide mode leads to a ray being emitted from the end face of the wedge structure and to a scattering from the tip apex.

Waveguide modes in a dielectric wedge are interpreted in terms of waveguide modes in an adiabatically tapered dielectric slab as a function of its thickness [2]. Figure 4 shows the dispersion relations of slab waveguide modes as a function of slab thickness. For all modes the wavenumber decreases with decreasing thickness of the slab. This property can be

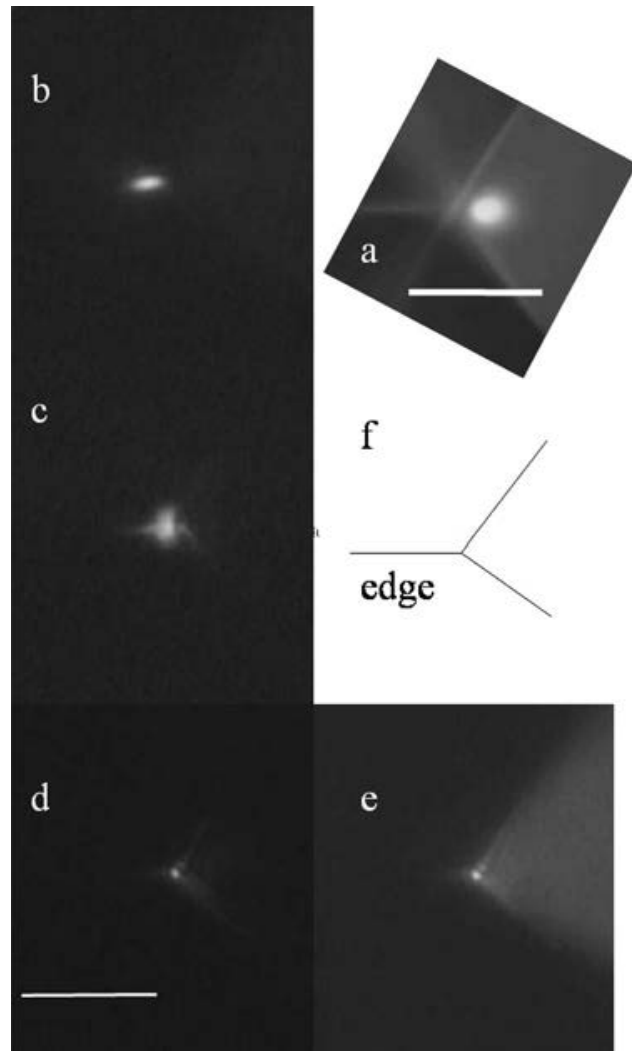

Fig. 3. Experimental observations. The white bars correspond to a $10 \mu \mathrm{m}$ (a) image of the reflected beam as recorded by CCD1 (see Fig. 2). To localize the focused spot in the tip structure, the tip was additionally illuminated by a weak nonlocal background illumination. (b) Image of the edge slightly displaced from the tip as recorded with CCD camera 2 (Fig. 2) showing light emission from the edge, which was illuminated by a high NA by adjusting iris diaphragm 2 (Fig. 2). (c) The same as (b) but with the tip in focus such that a blurred image of light emission from the edge and a weak emission from the tip is observed. (d) Image of the tip as in (b) but with a small NA of the illuminating beam such that no emission from the edge occurs. Only the emission from the tip is seen. The same NA of the exciting beam was chosen for image (a) of the reflected beam. (e) The same as (d) but with additional background illumination. (e) Schematic view of the orientation of the tip in the images.

described by an effective refractive index of the waveguide mode decreasing with decreasing slab thickness. We assume that in a wedge a waveguide mode exists at a certain height above its edge, which corresponds to the waveguide mode of a slab of a thickness corresponding to the width of the wedge at the given height. For a beam incident at an oblique angle into the wedge, the dispersion relations of Fig. 5 imply that the effective refractive index of the waveguide modes will continuously decrease as the beam penetrates into the wedge. This gradual decrease in the refractive index will lead to a gradual refraction and inclination of the incoming beam parallel to the edge. A similar phenomenon was discussed for an $s$-polarized surface plasmon mode in a metal-coated dielectric wedge [5]. There the effective refractive index increases with decreasing width within the edge. 


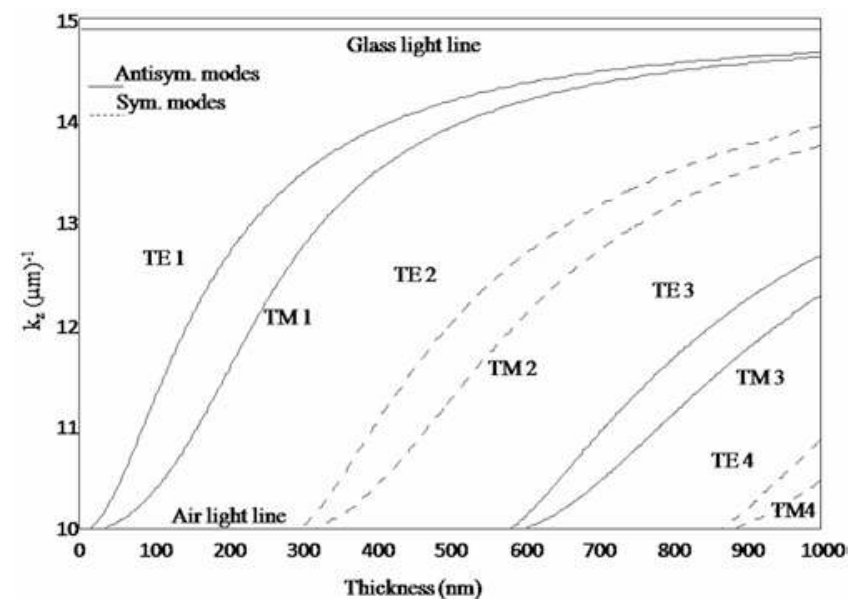

Fig. 4. (Color online) Dispersion relations as a function of the thickness of a dielectric slab for different symmetric and antisymmetric transverse electric (TE) and transverse magnetic (TM) modes for a vacuum wavelength of $630 \mathrm{~nm}$ showing a decrease in the wave vector $k_{z}$ with decreasing thickness of the slab in all cases.

Therefore the incoming beam is refracted toward the normal to the edge just opposite to the case of the dielectric wedge. For a $p$-polarized surface-plasmon mode, a very similar behavior is however expected as for the case of the purely dielectric wedge [6]. Figure 5 shows the result of numerical calculations by a volume integral equation method [6-8] of $x$ - and $y$-polarized Gaussian beams incident on a finite wedge at an angle of total reflection of $45^{\circ}$ with respect to the edge of the wedge. A standing wave phenomenon is seen parallel to the edge and emission of light at the tip. These numerical results give a detailed description of our experimental results.

Our results may be of interest for the development of sensors based on leaky wedge waveguide modes $[9,10]$. A wedge configuration would allow for a higher confinement of the sensing area than a planar configuration. The results are also of interest in the process of superfocusing in a metal-coated dielectric wedge by dimensional reduction in surface to edge plasmon modes as discussed recently [6]. Leaky waveguide modes as described here provide an efficient channel for the excitation of surface and edge plasmon modes in a metal-coated wedge as will be described explicitly in a forthcoming publication.

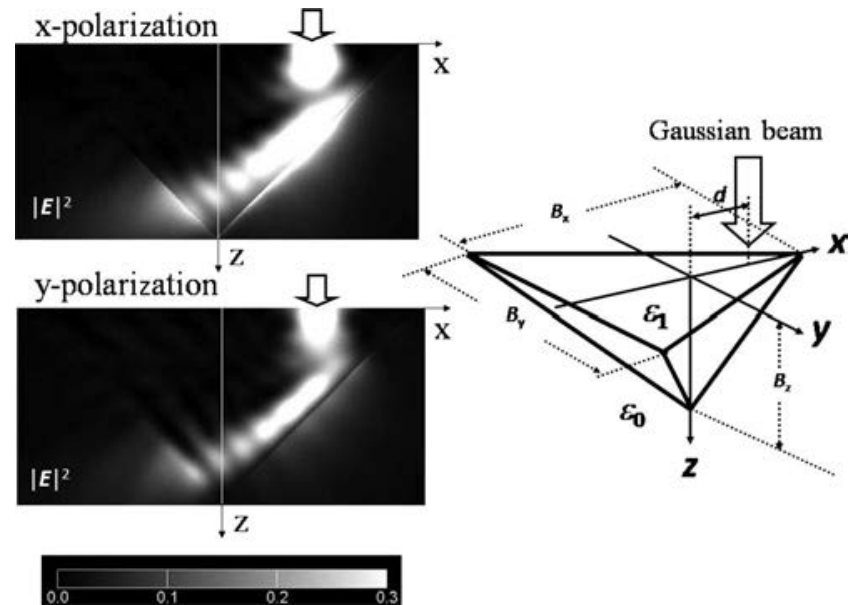

Fig. 5. Numerical results of the intensity of the electric field $\left|E^{2}\right|$ in a dielectric tip of $\varepsilon / \varepsilon_{0}=2.25$ surrounded by vacuum with permittivity $\varepsilon_{0}$ and of dimensions $B_{x}=6.1 \lambda$ and $B_{y}=8.15 \lambda$, where $\lambda$ is the vacuum wavelength. The spot size is $0.8 \lambda$, the intensity is unity at $z=0$, and the beam axis is in the $x-z$ plane parallel to the $z$ axis but displaced from it by a distance $d=1.42 \lambda$. The field intensity is shown for a section at $y=0$.

This work was supported by the German Science Foundation (DFG) (contract Fi:608/5-1) and by the Network of Excellence Plasmo-Nano-Devices in the FP6 IST program of the European Community.

\section{References}

1. B. R. Horowitz and T. Tamir, J. Opt. Soc. Am. 61, 586 (1971).

2. B. Polat, E. Topuz, and L. Sevgi, AEU, Int. J. Electron. Commun. 52, 105 (1998).

3. T. R. Matzelle, H. Gnaegi, A. Ricker, and R. Reichelt, J. Microsc. 209, 113 (2003).

4. U. C. Fischer, J. Koglin, and H. Fuchs, J. Microsc. 176, 231 (1994).

5. D. K. Gramotnev, J. Appl. Phys. 98, 104302 (2005).

6. K. Tanaka, G.W. Burr, T. Grosjean, T. Maletzky, and U. C. Fischer, Appl. Phys. B 93, 257 (2008).

7. P. Zwamborn and P. M. van den Berg, IEEE Trans. Microwave Theory Tech. 40, 1757 (1992).

8. K. Tanaka, M. Tanaka, and T. Sugiyama, Opt. Express 13, 256 (2005).

9. L. Kang and R. E. Dessy, Crit. Rev. Anal. Chem. 21, 377 (1990).

10. T. Hashimoto and T. Yoshino, Opt. Lett. 14, 913 (1989). 